\title{
Antimicrobial sensitivity patterns of cerebrospinal fluid (CSF) isolates in Namibia: implications for empirical antibiotic treatment of meningitis
}

Assegid Mengistu1*, Johannes Gaeseb', Gottfried Uaaka², Christophine Ndjavera², Kennedy Kambyambya', Lazarus Indongo ${ }^{1}$, Francis Kalemeera ${ }^{3}$, Christopher Ntege ${ }^{3}$, David Mabirizi ${ }^{3}$, Mohan P Joshi ${ }^{3}$ and Evans Sagwa ${ }^{3}$

\begin{abstract}
Objective: Bacterial meningitis is a medical emergency associated with high mortality rates. Cerebrospinal fluid (CSF) culture is the "gold standard" for diagnosis of meningitis and it is important to establish the susceptibility of the causative microorganism to rationalize treatment. The Namibia Standard Treatment Guidelines (STGs) recommends initiation of empirical antibiotic treatment in patients with signs and symptoms of meningitis after taking a CSF sample for culture and sensitivity. The objective of this study was to assess the antimicrobial sensitivity patterns of microorganisms isolated from CSF to antibiotics commonly used in the empirical treatment of suspected bacterial meningitis in Namibia.
\end{abstract}

Methods: This was a cross-sectional descriptive study of routinely collected antibiotic susceptibility data from the Namibia Institute of Pathology (NIP) database. Results of CSF culture and sensitivity from January 1, 2009 to May 31, 2012, from 33 state hospitals throughout Namibia were analysed.

Results: The most common pathogens isolated were Streptococcus species, Neisseria meningitidis, Haemophilus influenzae, Staphylococcus, and Escherichia coli. The common isolates from CSF showed high resistance (34.3\% 73.5\%) to penicillin. Over one third (34.3\%) of Streptococcus were resistance to penicillin which was higher than 24.8\% resistance in the United States. Meningococci were susceptible to several antimicrobial agents including penicillin. The sensitivity to cephalosporins remained high for Streptococcus, Neisseria, E. coli and Haemophilus. The highest percentage of resistance to cephalosporins was seen among ESBL K. pneumoniae $(n=7,71 \%-100 \%)$, other Klebsiella species ( $n=7,28 \%-80 \%)$, and Staphylococcus ( $n=36,25 \%-40 \%)$.

Conclusions: The common organisms isolated from CSF were Streptococcus Pneumoniae, Neisseria meningitidis, Haemophilus influenzae, Staphylococcus, and E. coli. All common organisms isolated from CSF showed high sensitivity to cephalosporins used in the empirical treatment of meningitis. The resistance of the common isolates to penicillin is high. Most ESBL K. pneumoniae were isolated from CSF samples drawn from neonates and were found to be resistant to the antibiotics recommended in the Namibia STGs. Based on the above findings, it is recommended to use a combination of aminoglycoside and third-generation cephalosporin to treat non-ESBL Klebsiella isolates. Carbapenems (e.g., meropenem) and piperacillin/tazobactam should be considered for treating severely ill patients with suspected ESBL Klebsiella infection. Namibia should have a national antimicrobial resistance surveillance system for early detection of antibiotics that may no longer be effective in treating meningitis and other life-threatening infections due to resistance.

Keywords: Celebrospinal fluid, Antimicrobial resistance, Culture and sensitivity, Empiric therapy, Meningitis, Namibia

\footnotetext{
* Correspondence: atmengistu@tipc.com.na

'Ministry of Health and Social Services, Windhoek, Namibia

Full list of author information is available at the end of the article
} 


\section{Introduction}

Bacterial meningitis is an acute infection in which the meninges, the subarachnoid space, and the brain parenchyma are all frequently involved in the inflammatory reaction. This disease is characterized by severe headache, fever, intolerance to light and sound and rigidity of muscles, especially those of the neck. The central nervous system (CNS) inflammatory reaction from bacterial meningitis may result in decreased consciousness, seizures, raised intracranial pressure, and stroke [1].

Bacterial meningitis is a medical emergency. The therapeutic goal is to initiate antibiotic therapy within 60 minutes of a patient's arrival in the emergency room. In patients suspected of having bacterial meningitis, cerebrospinal fluid (CSF) should be obtained for cultures and empirical antimicrobial therapy initiated without delay [2,3]. Diagnosis of bacterial meningitis is confirmed by CSF culture the "gold standard" for diagnosis of meningitis and it is equally important to obtain the antimicrobial susceptibility of the causative microorganism to rationalize treatment $[4,5]$.

The organisms most commonly responsible for community-acquired bacterial meningitis are Streptococcus pneumoniae, Neisseria meningitidis, Streptococci group B, Listeria monocytogenes, and Haemophilus influenza [6,7]. In children, meningococcal, Haemophilus influenzae type $\mathrm{b}$ (Hib), and pneumococcal infections are the most common causes [7].

Specific antibiotic treatment for bacterial meningitis depends upon identification of the causative organism. More than $80 \%$ of patients with common bacterial meningitis are culture positive. However, CSF culture positivity decreases with prior antibiotic treatment before lumbar puncture [4].

As infections of the CNS are potentially life threatening, empirical therapy should be initiated promptly whenever bacterial meningitis is a major diagnostic consideration. The Namibia Standard Treatment Guidelines (STGs) recommend initiation of empirical antibiotics treatment in patients with signs and symptoms of meningitis after taking a CSF sample for analysis, culture, and antibiotic sensitivity testing. The preferred medicines for patient with signs and symptoms of meningitis are based on knowledge of the common causative agent of meningitis in the specific patient group and the sensitivity of the suspected pathogen to the commonly used antimicrobials.

However, many infectious diseases are becoming increasingly difficult to treat because of antimicrobialresistant organisms. Both the epidemiology of bacterial meningitis and the sensitivity to antibiotics are changing as a result of the widespread use of antimicrobials and other factors [4]. Antimicrobial susceptibility data among CNS pathogens is therefore important to effectively manage meningitis patients in the first critical hours of their treatment [8]. Empirical antibiotic therapy should be adjusted to local drug resistance patterns and clinical subgroups. Accurate information regarding the important etiological agents and populations at risk is necessary to ascertain public health measures and ensure appropriate management of bacterial meningitis $[9,10]$.

It is therefore essential to monitor the emergence of resistance to antibiotics that are used for the empirical treatment as delay in providing effective treatment may adversely affect a patient's treatment outcome.

\section{Objectives}

- To determine the common microorganisms isolated from cerebrospinal fluid that are responsible for infectious meningitis in Namibia

- To establish the prevalence of antimicrobial sensitivity of microorganisms isolated from clinical cerebrospinal fluid samples in Namibia

- To determine the most appropriate medicines for the treatment of meningitis in Namibia

\section{Methods}

This was a cross-sectional descriptive study using routinely collected antibiotic susceptibility data from Namibia Institute of Pathology (NIP) database. Results of CSF culture and sensitivity from January 1, 2009 to May 31, 2012, from 33 out of 35 state hospitals throughout Namibia were stored in Meditech ${ }^{\circ}$. NIP uses this commercial laboratory management software to capture routinely collected antibiotic susceptibility and other laboratory data from public sector health facilities throughout the country. Cerebrospinal fluid clinical samples were transported and immediately processed at the NIP's regional or central laboratory. Standard CSF bacterial culture is accomplished using horse blood agar (5\%) or chocolate agar.

Pathogen identification and antibacterial susceptibilities were performed as appropriate using Wellcogen ${ }^{\circ}$ Bacterial Antigen Kit, Gram stain, and methylene blue stain. Standard antibiogram profile tests were done for specific microorganism isolates with some variations according to the doctors' request. Culture results were read after 24 hours of incubation. Plates were reincubated for a further 24 hours and re-examined for additional organisms. The results were recorded on a worksheet and entered into Meditech. Printed results were sent back to the clinicians who could also obtain the results by logging into the tool's web-based reporting module.

WHONET, a database software developed by the World Health Organization Collaborating Centre for Surveillance of Antimicrobial Resistance, was used to extract the data 
from all CSF samples. The extracted data was cleaned thorough visual checks and preliminary frequency counts on the raw data set and identified errors were corrected by the lead author. Cleaned data was analysed using Statistical Package for the Social Sciences ${ }^{\oplus}$ version 12.0.1.

The main outcome variable was the proportion of samples with positive cultures of suspected microorganisms. We also looked into the sensitivity patterns of isolates from CSF. Descriptive statistics were used to summarize the frequencies and distributions of microbial isolates and their sensitivity to various antimicrobials.

Since the analysis was conducted on de-identified electronic records of samples tested by the NIP, individual patient consent was not required. Authorization to conduct the analysis was granted by the Permanent Secretary of Ministry of Health and Social Services of Namibia (MoHSS) and the NIP management.

\section{Results}

A total of 7,267 CSF samples were tested at the NIP central and regional laboratories for culture and antimicrobial sensitivity. Out of the 7,267 CSF samples submitted, 701 (9.6\%) showed growth of microorganisms. Out of the 701 samples with growth of microorganism, 503 (71.8\%) grew bacteria, 188 (26.8\%) grew fungi (mainly Cryptococcus), and the remaining 10 samples (1.4\%) grew mycobacterium (Table 1).

The most frequent gram positive organisms isolated were Streptococcus species $(\mathrm{n}=206,40.9 \%)$, Staphylococcus $(\mathrm{n}=36,7.2 \%)$, and Enterococcus species $(\mathrm{n}=9,1.8 \%)$. The three most frequently identified gram negative organisms isolated were Neisseria meningitidis ( $N$. meningitidis) ( $\mathrm{n}=107,21.3 \%)$, Haemophilus influenzae $(\mathrm{n}=59,11.7 \%)$, and Escherichia coli (E. coli) $(\mathrm{n}=22$, 4.4\%) (Table 2).

Extended spectrum beta lactamase (ESBL) Klebsiella pneumoniae ( $K$. pneumonia) was the most frequently isolated microorganism in CSF samples collected from neonates (4/9) whereas Haemophilus, Streptococcus, and Staphylococcus were common in CSF samples collected from infants aged from one to 11 months. In the age group 1-5 years, Neisseria, Haemophilus, and Streptococcus were commonly isolated (Table 3). Neisseria and

Table 1 Type of organism isolated from CSF

\begin{tabular}{lrr}
\hline Type of organism & Frequency (\%) & Cumulative (\%) \\
\hline Gram Negative & $239(3.3 \%)$ & $239(3.3 \%)$ \\
Gram Positive & $264(3.6 \%)$ & $503(6.9 \%)$ \\
Fungi & $188(2.6 \%)$ & $691(9.5 \%)$ \\
Mycobacterium & $10(0.1 \%)$ & $701(9.6 \%)$ \\
No growth & $6,566(90.4 \%)$ & $7,267(100.0 \%)$ \\
Total & $7267(100.0 \%)$ & \\
\hline
\end{tabular}

Source: MEDITECH Database 2009-2012.
Streptococcus were commonly isolated in CSF samples drawn from the age group 6-12 years. In adults and adolescents 12 years and older, Neisseria, E. coli, Streptococcus, and Staphylococcus were the most frequent isolates (Table 3). The majority of the isolated bacteria $(n=409$, 81.3\%) were from inpatient CSF samples, followed by samples from the outpatient $(n=40,8.0 \%)$ and paediatrics departments ( $n=43,8.5 \%)$ (Table 4$)$.

We found that Streptococcus species were the most common gram positive organisms isolated from clinical CSF samples analyzed by NIP. Streptococci showed very high resistance to sulfamethoxazole/trimethoprim ( $\mathrm{n}=$ $125,71.4 \%)$ and high resistance to oxacillin $(n=69$, $45.1 \%)$, penicillin $(n=58,34.3 \%)$, and amoxicillin $(n=6$, 25.0\%). Streptococci were moderately resistant for gentamicin, tetracycline, clindamycin, and erythromycin, ranging from 12.4 to $20.0 \%$. The resistance to ceftriaxone, ofloxacin, cefuroxime, chloramphenicol, and vancomycin ranged from $2.2 \%$ to $5.5 \%$. All of the bacterial isolates $(\mathrm{n}=34,100.0 \%)$ that were tested for ciprofloxacin were found to be sensitive to this medicine (Table 5).

Among the gram negative bacteria, N.meningitidis was the most frequently isolated pathogen, and showed the following patterns of resistance sulfamethoxazole/trimethoprim ( $n=79,77.5 \%)$, erythromycin $(n=4,28.6 \%)$, penicillin $(n=16,15.2 \%)$, tetracycline $(n=3,12.5 \%)$, cefuroxime $(\mathrm{n}=5,4.8 \%)$, and chloramphenicol $(\mathrm{n}=3$, 2.9\%) (Table 5).

Haemophilus isolates were resistant to sulfamethoxazole/trimethoprim ( $\mathrm{n}=25,67.6 \%)$, amoxicillin $(\mathrm{n}=18$, $34 \%)$, cefuroxime $(\mathrm{n}=5,25 \%)$, amoxicillin/clavulanic acid $(n=4,22.2 \%)$, tetracycline $(n=4,14.8 \%)$, chloramphenicol ( $n=6,10.9 \%)$, and ceftriaxone $(n=5,9.6 \%)$. All Haemophilus isolates were sensitive to penicillin $\mathrm{G}$ and ciprofloxacin (Table 5).

Staphylococcus isolates showed relatively high resistance to most antibiotics ranging from $25.0 \%$ to $78.6 \%$. The highest rate of resistance was for amoxicillin $(\mathrm{n}=11$, $78.6 \%)$ followed by penicillin $(n=25,73.5 \%)$, sulfamethoxazole/trimethoprim $(n=18,62.1 \%)$, gentamicin $(n=9$, $52.9 \%)$ and cefuroxime $(\mathrm{n}=2,40 \%)$. The resistance to cloxacillin, oxacillin, ofloxacin, erythromycin, tetracycline, and cephalothin ranged between $34.5 \%-25 \%$. Staphylococcus were less resistant to fusidic acid ( $\mathrm{n}=1,2.9 \%)$. All staphylococcus isolates were found to be sensitive to vancomycin and amikacin (Table 5).

Most E. coli isolates were resistant to sulfamethoxazole/trimethoprim $(\mathrm{n}=18,85.7 \%)$ and amoxicillin $(\mathrm{n}=19$, 86.4\%). E. Coli showed resistance to piperacillin/tazobactam, tetracycline, amoxicillin/clavulanic acid, penicillin and erythromycin ranging between $25 \%-50 \%$. On the other hand, $E$. Coli showed lower rates of resistance to gentamicin $(\mathrm{n}=3,17.65 \%)$, cefuroxime $(\mathrm{n}=2,10.5 \%)$, cephalothin $(\mathrm{n}=1,10 \%)$, and ciprofloxacin $(\mathrm{n}=1,7.7 \%)$. All E. Coli 
Table 2 Bacteria isolated from CSF

\begin{tabular}{|c|c|c|c|c|}
\hline Organism isolate & $\begin{array}{l}\text { Frequency } \\
\text { of isolate }\end{array}$ & $\%$ & $\begin{array}{l}\text { Cumulative } \\
\text { frequency }\end{array}$ & $\begin{array}{c}\text { Cumulative } \\
\%\end{array}$ \\
\hline $\begin{array}{l}\text { Streptococcus } \\
\text { pneumoniae }\end{array}$ & 187 & 37.18 & 187 & 37.18 \\
\hline $\begin{array}{l}\text { Neisseria meningitids } \\
\text { group A }\end{array}$ & 53 & 10.54 & 240 & 47.71 \\
\hline Neisseria meningitidis & 48 & 9.54 & 288 & 57.26 \\
\hline $\begin{array}{l}\text { Haemophilus } \\
\text { influenzae }\end{array}$ & 38 & 7.55 & 326 & 64.81 \\
\hline Escherichia coli & 22 & 4.37 & 348 & 69.18 \\
\hline $\begin{array}{l}\text { Staphylococcus } \\
\text { aureus }\end{array}$ & 19 & 3.78 & 367 & 72.96 \\
\hline Haemophilus species & 15 & 2.98 & 382 & 75.94 \\
\hline $\begin{array}{l}\text { Staphylococcus } \\
\text { epidermides }\end{array}$ & 10 & 1.99 & 392 & 77.93 \\
\hline $\begin{array}{l}\text { Streptococcus } \\
\text { species }\end{array}$ & 10 & 1.99 & 402 & 79.92 \\
\hline Enterococcus faecalis & 7 & 1.39 & 409 & 81.31 \\
\hline $\begin{array}{l}\text { ESBL Klebsiella } \\
\text { pneumoniae }\end{array}$ & 7 & 1.39 & 416 & 82.7 \\
\hline $\begin{array}{l}\text { Haemophilus } \\
\text { influenzae type B }\end{array}$ & 6 & 1.19 & 422 & 83.9 \\
\hline $\begin{array}{l}\text { Pseudomonas } \\
\text { aeruginosa }\end{array}$ & 5 & 0.99 & 427 & 84.89 \\
\hline $\begin{array}{l}\text { Streptococcus group } \\
\text { B }\end{array}$ & 4 & 0.8 & 431 & 85.69 \\
\hline $\begin{array}{l}\text { Acinetobacter } \\
\text { baumanii }\end{array}$ & 4 & 0.8 & 435 & 86.48 \\
\hline $\begin{array}{l}\text { Klebsiella } \\
\text { pneumoniae }\end{array}$ & 4 & 0.8 & 439 & 87.28 \\
\hline Bacillus cereus & 3 & 0.6 & 442 & 87.87 \\
\hline Micrococcus species & 3 & 0.6 & 445 & 88.47 \\
\hline Gram negative bacilli & 3 & 0.6 & 448 & 89.07 \\
\hline Serratia liquifaciens & 3 & 0.6 & 451 & 89.66 \\
\hline Gram positive cocci & 2 & 0.4 & 453 & 90.06 \\
\hline $\begin{array}{l}\text { Listeria } \\
\text { monocygenes }\end{array}$ & 2 & 0.4 & 455 & 90.46 \\
\hline $\begin{array}{l}\text { Staphylococcus } \\
\text { capitis }\end{array}$ & 2 & 0.4 & 457 & 90.85 \\
\hline $\begin{array}{l}\text { Staphylococcus } \\
\text { haemolyticus }\end{array}$ & 2 & 0.4 & 459 & 91.25 \\
\hline $\begin{array}{l}\text { Streptococcus group } \\
\text { A }\end{array}$ & 2 & 0.4 & 461 & 91.65 \\
\hline $\begin{array}{l}\text { Streptococcus } \\
\text { viridans group }\end{array}$ & 2 & 0.4 & 463 & 92.05 \\
\hline Acinetobacter Iwoffii & 2 & 0.4 & 465 & 92.45 \\
\hline $\begin{array}{l}\text { ESBL's Escherichia } \\
\text { coli }\end{array}$ & 2 & 0.4 & 467 & 92.84 \\
\hline Klebsiella oxytoca & 2 & 0.4 & 469 & 93.24 \\
\hline $\begin{array}{l}\text { Neisseria meningitidis } \\
\text { group C }\end{array}$ & 2 & 0.4 & 471 & 93.64 \\
\hline $\begin{array}{l}\text { Neisseria meningitidis } \\
\text { W135 }\end{array}$ & 2 & 0.4 & 473 & 94.04 \\
\hline
\end{tabular}

Table 2 Bacteria isolated from CSF (Continued)

\begin{tabular}{|c|c|c|c|c|}
\hline Neisseria species & 2 & 0.4 & 475 & 94.43 \\
\hline Salmonella species & 2 & 0.4 & 477 & 94.83 \\
\hline Serratia marcescens & 2 & 0.4 & 479 & 95.23 \\
\hline $\begin{array}{l}\text { Sphingomonas } \\
\text { paucimobilis }\end{array}$ & 2 & 0.4 & 481 & 95.63 \\
\hline Bacillus species & 1 & 0.2 & 482 & 95.83 \\
\hline $\begin{array}{l}\text { Diphtheroid } \\
\text { organisms }\end{array}$ & 1 & 0.2 & 483 & 96.02 \\
\hline $\begin{array}{l}\text { Enterococcus } \\
\text { faecium }\end{array}$ & 1 & 0.2 & 484 & 96.22 \\
\hline Enterococcus species & 1 & 0.2 & 485 & 96.42 \\
\hline Kocuria varians & 1 & 0.2 & 486 & 96.62 \\
\hline $\begin{array}{l}\text { Staphylococcus } \\
\text { hominis }\end{array}$ & 1 & 0.2 & 487 & 96.82 \\
\hline $\begin{array}{l}\text { Staphylococcus } \\
\text { saprophyticus }\end{array}$ & 1 & 0.2 & 488 & 97.02 \\
\hline $\begin{array}{l}\text { Staphylococcus } \\
\text { simulans }\end{array}$ & 1 & 0.2 & 489 & 97.22 \\
\hline $\begin{array}{l}\text { Streptococcus } \\
\text { agalacteae }\end{array}$ & 1 & 0.2 & 490 & 97.42 \\
\hline Acinetobacter junii & 1 & 0.2 & 491 & 97.61 \\
\hline $\begin{array}{l}\text { Acinetobacter } \\
\text { species }\end{array}$ & 1 & 0.2 & 492 & 97.81 \\
\hline $\begin{array}{l}\text { Aeromonas } \\
\text { hydrophilia }\end{array}$ & 1 & 0.2 & 493 & 98.01 \\
\hline Coliform bacillus & 1 & 0.2 & 494 & 98.21 \\
\hline Enterobacter species & 1 & 0.2 & 495 & 98.41 \\
\hline $\begin{array}{l}\text { Haemophilus } \\
\text { parainfluenzae }\end{array}$ & 1 & 0.2 & 496 & 98.61 \\
\hline Klebsiella ozaenae & 1 & 0.2 & 497 & 98.81 \\
\hline Moraxella species & 1 & 0.2 & 498 & 99.01 \\
\hline Morganella morganii & 1 & 0.2 & 499 & 99.2 \\
\hline $\begin{array}{l}\text { Pseudomonas } \\
\text { paucimobilis }\end{array}$ & 1 & 0.2 & 500 & 99.4 \\
\hline $\begin{array}{l}\text { Pseudomonas } \\
\text { species }\end{array}$ & 1 & 0.2 & 501 & 99.6 \\
\hline $\begin{array}{l}\text { Salmonella } \\
\text { omnivalent positive }\end{array}$ & 1 & 0.2 & 502 & 99.8 \\
\hline Yersinia pestis & 1 & 0.2 & 503 & 100 \\
\hline
\end{tabular}


Table 3 Frequency of organisms isolates by age group

\begin{tabular}{|c|c|c|c|c|c|c|c|c|}
\hline Organism & $<1 \mathrm{mo}$ & 1-11 mos & $1-55$ yrs & $6-12$ yrs & $>12$ yrs & Age Unk & Total & $\%$ of total \\
\hline Streptococcus pneumoniae & 2 & 31 & 27 & 17 & 87 & 42 & 187 & 37.18 \\
\hline Neisseria species & 2 & 5 & 14 & 18 & 56 & 12 & 107 & 21.27 \\
\hline Haemophilus species & 0 & 18 & 24 & 2 & 9 & 6 & 59 & 11.73 \\
\hline Staphylococcus & 0 & 10 & 6 & 2 & 11 & 7 & 36 & 7.16 \\
\hline Others Gram negative & 0 & 11 & 2 & 0 & 7 & 2 & 22 & 4.39 \\
\hline Escherichia coli & 1 & 2 & 1 & 0 & 15 & 3 & 22 & 4.37 \\
\hline Streptococcus species & & & & & & & 19 & 3.77 \\
\hline Other Gram positives & 0 & 4 & 3 & 1 & 5 & 0 & 13 & 2.6 \\
\hline Enterococcus species & 0 & 3 & 0 & 2 & 1 & 3 & 9 & 1.79 \\
\hline Acinetobacter species & 0 & 4 & 0 & 2 & 2 & 0 & 8 & 1.59 \\
\hline ESBL's Klebsiella pneumoniae & 4 & 2 & 0 & 0 & 0 & 1 & 7 & 1.39 \\
\hline Klebsiella & 0 & 1 & 1 & 0 & 4 & 1 & 7 & 1.39 \\
\hline Pseudomonas species & 0 & 2 & 2 & 1 & 2 & 0 & 7 & 1.39 \\
\hline Total & 9 & 93 & 80 & 45 & 199 & 77 & 503 & 100.02 \\
\hline
\end{tabular}

Source: MEDITECH Database 2009-2012. Legend: mo=month; mos=months; yrs=years; age unk= age unknown; \% = percent.

isolates $(100 \%)$ were sensitive to ceftriaxone and chloramphenicol (Table 5).

Klebsiella isolates were resistant to cephalothin $(\mathrm{n}=4$, $80.0 \%)$, amoxicillin $(\mathrm{n}=4,57.1 \%)$, sulfamethoxazole/trimethoprim $(n=4,57.1 \%)$, cefuroxime $(n=2,33.3 \%)$, and piperacillin/tazobactam $(\mathrm{n}=1,33.3 \%)$. All Klebsiella $(\mathrm{n}=5$, $100 \%)$ showed sensitivity to ciprofloxacin. However, the ESBL-K. pneumonia isolates $(\mathrm{n}=7)$ were resistant to nearly all commonly used antibiotics such as amoxicillin, ceftriaxone, cefuroxime, gentamicin, and sulfamethoxazole/trimethoprim (Table 5).

\section{Discussion}

The study was aimed at determining the antimicrobial sensitivity patterns of common microorganisms isolated from clinical samples of CSF and to recommend appropriate medicines for the empirical treatment of meningitis in Namibia.

There are several laboratory tests that are useful in the diagnosis of central nervous system infection, yet no single laboratory test or clinical feature can distinguish between different types of central nervous system infections. Some clinicians propose clinical decision rules which combine

Table 4 Frequency of organisms isolated by hospital department

\begin{tabular}{|c|c|c|c|c|c|}
\hline Organism & Inpatient & Outpatient & Pediatrics & Missing & Total \\
\hline Streptococcus species & 162 & 16 & 19 & 9 & 206 \\
\hline Neisseria species & 94 & 11 & 2 & 0 & 107 \\
\hline Haemophilus species & 47 & 2 & 9 & 1 & 59 \\
\hline Staphylococcus aureus & 34 & 1 & 1 & 0 & 36 \\
\hline Escherichia coli & 13 & 7 & 1 & 1 & 22 \\
\hline Other Gram negative & 20 & 2 & 0 & 0 & 22 \\
\hline Other Gram positive & 12 & 0 & 1 & 0 & 13 \\
\hline Enterococcus species & 8 & 0 & 1 & 0 & 9 \\
\hline Acinetobacter species & 6 & 0 & 2 & 0 & 8 \\
\hline ESBL's Klebsiella pneumoniae & 6 & 1 & 0 & 0 & 7 \\
\hline Klebsiella & 7 & 0 & 0 & 0 & 7 \\
\hline Pseudomonas species & 0 & 0 & 7 & 0 & 7 \\
\hline Total & 409 (81.3\%) & 40 (8.0\%) & $43(8.5 \%)$ & $11(2.2 \%)$ & $503(100 \%)$ \\
\hline
\end{tabular}

Source: MEDITECH Database 2009-2012. 
Table 5 Susceptibility of organisms isolated from CSF

\begin{tabular}{|c|c|c|c|c|c|c|c|c|}
\hline Organism & Antimicrobial & Total, N & $\mathrm{S}$ & $\mathrm{S}, \%$ & $\mathbf{R}$ & $\mathrm{R}, \%$ & I & I, \% \\
\hline \multirow[t]{14}{*}{ Streptococcus 206 isolates } & Ciprofloxacin & 34 & 34 & 100 & 0 & 0 & & 0 \\
\hline & Ceftriaxone & 89 & 87 & 97.8 & 2 & 2.2 & 0 & 0 \\
\hline & Ofloxacin & 40 & 39 & 97.5 & 1 & 2.5 & 0 & 0 \\
\hline & Cefuroxime & 28 & 27 & 96.4 & 1 & 3.6 & 0 & 0 \\
\hline & Chloramphenicol & 171 & 160 & 93.6 & 9 & 5.3 & 2 & 1.2 \\
\hline & Vancomycin & 110 & 102 & 92.7 & 6 & 5.5 & 2 & 1.8 \\
\hline & Erythromycin & 186 & 162 & 87.1 & 23 & 12.4 & 1 & 0.5 \\
\hline & Clindamycin & 140 & 120 & 85.7 & 19 & 13.6 & 1 & 0.7 \\
\hline & Tetracycline & 180 & 146 & 81.1 & 25 & 13.9 & 9 & 5 \\
\hline & Gentamicin & 5 & 4 & 80 & 1 & 20 & 0 & 0 \\
\hline & Amoxicillin & 24 & 18 & 75 & 6 & 25 & 0 & 0 \\
\hline & Penicillin & 169 & 109 & 64.5 & 58 & 34.3 & 2 & 1.2 \\
\hline & Oxacillin & 153 & 79 & 51.6 & 69 & 45.1 & 5 & 3.3 \\
\hline & Sulfamethoxazole/trimethoprim & 175 & 48 & 27.4 & 125 & 71.4 & 2 & 1.1 \\
\hline \multirow[t]{6}{*}{ Neisseria meningitidis 107 isolates } & Chloramphenicol & 102 & 97 & 95.1 & 3 & 2.9 & 2 & 2 \\
\hline & Ceftriaxone & 105 & 97 & 92.4 & 5 & 4.8 & 3 & 2.9 \\
\hline & Penicillin & 105 & 86 & 81.9 & 16 & 15.2 & 3 & 2.9 \\
\hline & Erythromycin & 14 & 10 & 71.4 & 4 & 28.6 & 0 & 0 \\
\hline & Tetracycline & 24 & 17 & 70.8 & 3 & 12.5 & 4 & 16.7 \\
\hline & Sulfamethoxazole/trimethoprim & 102 & 22 & 21.6 & 79 & 77.5 & 1 & 1 \\
\hline \multirow[t]{10}{*}{ Haemophilus 59 isolates } & Amoxicillin & 53 & 32 & 60.4 & 18 & 34 & 3 & 5.7 \\
\hline & Amoxicillin/Clavulanic Acid & 18 & 14 & 77.8 & 4 & 22.2 & & 0 \\
\hline & Ceftriaxone & 52 & 46 & 88.5 & 5 & 9.6 & 1 & 1.9 \\
\hline & Cefuroxime & 20 & 14 & 70 & 5 & 25 & 1 & 5 \\
\hline & Chloramphenicol & 55 & 48 & 87.3 & 6 & 10.9 & 1 & 1.8 \\
\hline & Ciprofloxacin & 11 & 11 & 100 & 0 & 0 & & 0 \\
\hline & Ofloxacin & 23 & 22 & 95.7 & 0 & 0 & 1 & 4.3 \\
\hline & Penicillin & 6 & 6 & 100 & & 0 & & 0 \\
\hline & Tetracycline & 27 & 21 & 77.8 & 4 & 14.8 & 2 & 7.4 \\
\hline & Sulfamethoxazole/trimethoprim & 37 & 10 & 27 & 25 & 67.6 & 2 & 5.4 \\
\hline \multirow[t]{13}{*}{ Staphylococcus 36 isolates } & Amikacin & 7 & 7 & 0 & 0 & 0 & 0 & 0 \\
\hline & Vancomycin & 22 & 20 & 90.9 & 0 & 0 & 2 & 9.1 \\
\hline & Fusidic acid & 34 & 29 & 85.3 & 1 & 2.9 & 4 & 11.8 \\
\hline & Ciprofloxacin & 21 & 17 & 81 & 4 & 19 & & 0 \\
\hline & Cephalothin & 8 & 6 & 75 & 2 & 25 & 0 & 0 \\
\hline & Clindamycin & 27 & 19 & 70.4 & 6 & 22.2 & 2 & 7.4 \\
\hline & Tetracycline & 27 & 19 & 70.4 & 8 & 29.6 & & 0 \\
\hline & Erythromycin & 31 & 21 & 67.7 & 10 & 32.3 & 0 & 0 \\
\hline & Oxacillin & 9 & 6 & 66.7 & 3 & 33.3 & 0 & 0 \\
\hline & Cloxacillin & 29 & 19 & 65.5 & 10 & 34.5 & & 0 \\
\hline & Cefuroxime & 5 & 3 & 60 & 2 & 40 & 0 & 0 \\
\hline & Gentamicin & 17 & 7 & 41.2 & 9 & 52.9 & 1 & 5.9 \\
\hline & Penicillin & 34 & 8 & 23.5 & 25 & 73.5 & 1 & 2.9 \\
\hline
\end{tabular}


Table 5 Susceptibility of organisms isolated from CSF (Continued)

\begin{tabular}{|c|c|c|c|c|c|c|c|c|}
\hline & Amoxicillin & 14 & 3 & 21.4 & 11 & 78.6 & 0 & 0 \\
\hline & Sulfamethoxazole/trimethoprim & 29 & 0 & 0 & 18 & 62.1 & 11 & 37.9 \\
\hline \multirow[t]{13}{*}{ Escherichia coli 22 isolates } & Ceftriaxone & 17 & 17 & 100 & & 0 & & 0 \\
\hline & Chloramphenicol & 6 & 6 & 100 & & 0 & & 0 \\
\hline & Ciprofloxacin & 13 & 12 & 92.3 & 1 & 7.7 & & 0 \\
\hline & Cefuroxime & 19 & 15 & 78.9 & 2 & 10.5 & 2 & 10.5 \\
\hline & Piperacillin-Tazobactam & 4 & 3 & 75 & 1 & 25 & & 0 \\
\hline & Cephalothin & 10 & 7 & 70 & 1 & 10 & 2 & 20 \\
\hline & Gentamicin & 17 & 11 & 64.71 & 3 & 17.65 & 3 & 17.65 \\
\hline & Tetracycline & 5 & 3 & 60 & 2 & 40 & & 0 \\
\hline & Amoxicillin/Clavulanic Acid & 7 & 4 & 57.14 & 3 & 42.86 & & 0.0 \\
\hline & Erythromycin & 4 & 2 & 50 & 2 & 50 & & 0 \\
\hline & Penicillin & 4 & 2 & 50 & 2 & 50 & & 0 \\
\hline & Sulfamethoxazole/trimethoprim & 21 & 3 & 14.29 & 18 & 85.71 & & 0 \\
\hline & Amoxicillin & 22 & 3 & 13.6 & 19 & 86.4 & & 0 \\
\hline \multirow[t]{8}{*}{ Enterococcus species 9 isolates } & Penicillin & 8 & 7 & 87.5 & 1 & 12.5 & 0 & 0 \\
\hline & Vancomycin & 9 & 7 & 77.8 & 1 & 11.1 & 1 & 11.1 \\
\hline & Amoxicillin & 7 & 5 & 71.4 & 2 & 28.6 & 0 & 0 \\
\hline & Ciprofloxacin & 3 & 2 & 66.7 & 1 & 33.3 & & 0 \\
\hline & Erythromycin & 3 & 1 & 33.3 & 2 & 66.7 & 0 & 0 \\
\hline & Clindamycin & 8 & 2 & 25 & 6 & 75 & 0 & 0 \\
\hline & Tetracycline & 2 & 0 & 0 & 2 & 100 & 0 & 0 \\
\hline & Sulfamethoxazole/trimethoprim & 2 & 0 & 0 & 2 & 100 & 0 & 0 \\
\hline \multirow[t]{6}{*}{ Acinetobacter species 8 isolates } & Imipenem & 8 & 7 & 87.5 & 1 & 12.5 & & 0 \\
\hline & Sulfamethoxazole/trimethoprim & 7 & 6 & 85.7 & 1 & 14.3 & & 0 \\
\hline & Gentamycin & 8 & 6 & 75 & 2 & 25 & 0 & 0 \\
\hline & Piperacillin-Tazobactam & 8 & 6 & 75 & 1 & 12.5 & 1 & 12.5 \\
\hline & Ceftazidime & 6 & 4 & 66.7 & 1 & 16.7 & 1 & 16.7 \\
\hline & Piperacillin & 2 & 1 & 50 & 1 & 50 & & 0 \\
\hline \multirow[t]{9}{*}{ Klebsiella 7 isolates } & Ciprofloxacin & 5 & 5 & 100 & & 0 & & 0 \\
\hline & Amikacin & 6 & 5 & 83.3 & 1 & 16.7 & & 0 \\
\hline & Ceftriaxone & 7 & 5 & 71.4 & 2 & 28.6 & & 0 \\
\hline & Gentamicin & 6 & 4 & 66.7 & 1 & 16.7 & 1 & 16.7 \\
\hline & Piperacillin-Tazobactam & 3 & 2 & 66.7 & 1 & 33.3 & & 0 \\
\hline & Cefuroxime & 6 & 3 & 50 & 2 & 33.3 & 1 & 16.7 \\
\hline & Sulfamethoxazole/trimethoprim & 7 & 3 & 42.9 & 4 & 57.1 & & 0 \\
\hline & Cephalothin & 5 & 1 & 20 & 4 & 80 & 0 & 0 \\
\hline & Amoxicillin & 7 & 1 & 14.3 & 4 & 57.1 & 2 & 28.6 \\
\hline \multirow[t]{7}{*}{ ESBL's Klebsiella pneumoniae 7 isolates } & Amikacin & 7 & 5 & 71.43 & 1 & 14.3 & 1 & 14.3 \\
\hline & Ciprofloxacin & 7 & 7 & 100 & & 0 & & 0 \\
\hline & Imipenem & 7 & 7 & 100 & & 0 & & 0 \\
\hline & Piperacillin-Tazobactam & 7 & 7 & 100 & & 0 & & 0 \\
\hline & Ceftazidime & 7 & 2 & 28.6 & 5 & 71.4 & & 0 \\
\hline & Amoxicillin & 7 & & 0 & 7 & 100 & & 0 \\
\hline & Ceftriaxone & 7 & & 0 & 7 & 100 & & 0 \\
\hline
\end{tabular}


Table 5 Susceptibility of organisms isolated from CSF (Continued)

\begin{tabular}{|c|c|c|c|c|c|c|c|c|}
\hline & Cefuroxime & 7 & & 0 & 7 & 100 & & 0 \\
\hline & Gentamicin & 7 & & 0 & 7 & 100 & & 0 \\
\hline & Sulfamethoxazole/trimethoprim & 7 & & 0 & 7 & 100 & & 0 \\
\hline \multirow[t]{7}{*}{ Pseudomonas species 7 isolates } & Amikacin & 7 & 7 & 100 & & 0 & & 0 \\
\hline & Ceftazidime & 7 & 7 & 100 & & 0 & & 0 \\
\hline & Ciprofloxacin & 6 & 6 & 100 & & 0 & & 0 \\
\hline & Imipenem & 6 & 6 & 100 & & 0 & & 0 \\
\hline & Gentamycin & 6 & 5 & 83.3 & 1 & 16.7 & 0 & 0 \\
\hline & Piperacillin-Tazobactam & 7 & 4 & 57.1 & & 0 & 3 & 42.9 \\
\hline & Sulfamethoxazole/trimethoprim & 7 & 3 & 42.9 & 4 & 57.1 & & 0 \\
\hline
\end{tabular}

Source: MEDITECH Database 2009-2012. legend: $S=$ sensitive; $R=$ resistant and I=indeterminate.

clinical and simple laboratory features. However, microbial culture and identification remain the gold standard for diagnosing bacterial meningitis $[11,12]$.

The etiology of central nervous system infections differs from place to place and with different age groups $[13,14]$. Studies have shown that bacterial meningitis is responsible for about $30 \%-40 \%$ of central nervous system infections. The remaining $60-70 \%$ are due to other etiologies such as viral meningitis, cryptococcal meningitis, brain abscess, tuberculosis meningitis, tuberculoma and others $[13,15]$.

In this analysis, $9.6 \%$ of the CSF samples showed microorganism growth. Studies have shown that CSF cultures are expected to be positive in $70 \%-85 \%$ of patients with bacterial meningitis who had no prior antimicrobial therapy $[4,15,16]$. In the present study, prior antibiotic treatment and high aseptic meningitis associated with HIV may have contributed to the low bacteria growth $[4,16]$. Autoimmune processes, HIV itself, and meningitis caused by fungal infections that occur in patients with late stage HIV infection can present with signs and symptom of meningitis with negative CSF culture [17].

The most common pathogens causing meningitis that were isolated from the CSF samples analysed by NIP were Streptococcus species, N. meningitidis, Haemophilus influenzae, Staphylococcus, and E. coli. All common organisms isolated from CSF the samples showed high resistance to penicillin. The $34.3 \%$ resistance of Streptococcus species to penicillin is much higher than the $24.8 \%$ resistance in the United States [18]. This is surprising as resistance rates are generally expected to be higher in developed countries [19].

The sensitivity to cephalosporins remained high for Streptococcus, Neisseria, E. coli, and Haemophilus. The 96.4\% sensitivity of S. pneumoniae for cefuroxime was comparable with $91.4 \%$ sensitivity in the United States [20] The highest percentage of resistance to a cephalosporin was seen among ESBL K. pneumoniae, Klebsiella, and Staphylococcus.
The profile of isolated organisms differed by age group. Staphylococcus seemed to be the common cause of meningitis in all age groups. Staphylococcus was moderately sensitive $(60 \%-75 \%)$ to the cephalosporins (cefuroxime, cephalothin) but it showed less sensitivity to penicillins $(23.5 \%)$ and zero sensitivity to sulfamethoxazole/trimethoprim which is not used in the empirical treatment of patients with suspected meningitis.

ESBL $K$. pneumoniae was the most frequent isolate in CSF samples drawn from neonates (4/9). This finding is different from the known common pathogens that cause meningitis during the first week of life in the United States [4]. There has been a significant increase in ESBL Klebsiella species reported in recent years [21,22]. These strains are highly virulent and have an extraordinary ability to spread, and can result in bacteremia and significantly increase mortality. Klebsiellae have been incriminated in nosocomial infections [21,22]. ESBL Klebsiella has become a major problem in hospitals because of resistance to multiple antibiotics [22]. In addition to meningitis, it can cause pneumonia, bacteremia, thrombophlebitis, urinary tract infection, cholecystitis, diarrhea, upper respiratory tract infection, wound infection, osteomyelitis, and meningitis.

The medical use of invasive devices, contamination of respiratory support equipment and use of antibiotics are factors that increase the likelihood of nosocomial infection with Klebsiella species. Sepsis and septic shock may follow entry of organisms into the blood from a focal source. The Namibia STGs recommend empirical treatment of suspected meningitis in children younger than three months with amoxicillin combined with gentamicin as first choice and ceftriaxone as alternative. Yet, the ESBL's Klebsiella isolate was found to be sensitive to ciprofloxacin, imipenem, and piperacillin/tazobactam and resistant to almost all the medicines included in the Namibia STGs, such as amoxicillin and gentamicin.

Most of the ESBL K. pneumoniae were isolated from CSF samples drawn from neonates. Haemophilus species were common isolate in one month to 11 months and 
1-5 years age groups. Haemophilus, Streptococcus, and Staphylococcus were common in CSF samples drawn from infants aged from one month to 11 months whereas Streptococcus, Neisseria, and Haemophilus were commonly isolated in the 1-5 years age group. In the 6-12 years age group, Neisseria and Streptococcus were the most common isolate. Streptococcus pneumoniae, Neisseria specie, Escherichia coli and Staphylococcus were common isolate in adults. Streptococcus pneumoniae and Neisseria specie were common isolate in all age group and were found to be sensitive to cephalosporins recommended in the STGs (ceftriaxone and cefuroxime). However, $40.0 \%$ of Staphylococci and 25.0\% of Haemophilus isolates were resistant to cefuroxime. Staphylococcus and Haemophilus species were also found to be resistant to amoxicillin in $78.6 \%$ and $34.0 \%$ of the cases. Staphylococci and Haemophilus were second and third common isolate in infants aged one month to 11 months following Streptococcus pneumoniae. Other studies have indicated that $80 \%$ of community-acquired bacterial meningitis in children aged three months and older are due to $S$. pneumoniae and $N$. meningitidis and empirical coverage with cephalosporin (cefuroxime or ceftriaxone) is recommended [10].

In adults and adolescents, Streptococcus and Neisseria were the most frequent isolates-this is consistent with the finding of other similar studies [10]. Both have high sensitivity to ceftriaxone.

\section{Strengths and implications}

The strength of this epidemiologic analysis is that it provided the national picture of microorganisms causing CNS infection and their resistance pattern. It also showed the common isolate among different age group and their resistance pattern. These finding can be used to guide empirical treatment of patients with suspected meningitis. It can also inform the review of treatment guidelines. This exercise demonstrated the importance of analyzing routinely collected clinical laboratory data in the monitoring of the emergence of antimicrobial resistance. The analysis was time efficient and inexpensive. The analysis can be replicated on other clinical conditions to identify the common causative organism or repeated at a later date to see trends in antimicrobial resistance patterns.

\section{Limitations}

The main limitation of this analysis was the use of secondary data. The data was not primarily collected to answer a specific a priori research question but as part of NIP's routine testing clinical samples. The laboratory tests' results are recorded mainly for reporting back to clinicians as well for administrative and billing purposes. As a result of this set up, not all species of microbes were identified or tested against the antibiotics of interest. There were also some important variables not captured in the system, including the possible alternative diagnoses and a patient's history of prior treatment with antibiotics before collection the CSF samples.

\section{Conclusions}

The common organisms isolated from CSF samples submitted to the NIP were Streptococcus Pneumoniae, Neisseria meningitidis, Haemophilus influenzae, Staphylococcus, and E. coli. All common organisms isolated from CSF showed high sensitivity to the cephalosporins used in the empirical treatment of meningitis in Namibia. The resistance of common isolates to penicillin which is alternative to cephalosporins in the STG is high.

Most ESBL $K$. pneumoniae isolated from CSF samples were from neonates and were found to be resistant to the antibiotics recommended in the Namibia STGs for treating meningitis in children aged three months or younger. Based on the above findings, it is recommended to use a combination of aminoglycoside and thirdgeneration cephalosporin to treat non-ESBL Klebsiella isolates. Carbapenems (e.g., meropenem) and piperacillin/ tazobactam should be considered in severely ill patients with possible ESBL Klebsiella infection.

There is a need to strengthen the infection control practices in the public hospitals in Namibia. It is also recommended that a national antimicrobial resistance surveillance system be developed for early detection of resistance. This would be expected to help identify appropriate antibiotics for the management of meningitis in Namibia.

\section{Competing interests}

None of the authors have any competing interests to disclose.

\section{Authors' contributions}

AM analyzed the data, interpreted the results, and drafted and finalized the manuscript. GU and CN assisted in extracting and cleaning the data. JG, DM, $\mathrm{FK}$, and $\mathrm{CN}$ reviewed the protocol for the analysis and the final draft of the manuscript. KK, LI, and MPJ reviewed the final draft of the manuscript. ES guided the design of the analysis, provided input into the interpretation of the results, and critically reviewed all drafts of the manuscript. All authors read and approved the final manuscript.

\section{Acknowledgment}

The authors would like to express their utmost gratitude to the MoHSS for supporting and guiding this activity. We extend our special thanks to Ms. T. K. Angula, former Chief Executive Officer of the Namibia NIP, and Mr. H. Kaura, General Manager Technical Operations and acting Chief Executive Officer of the NIP for their support in making the Meditech database available. We also thank Mr. Victor Sumbi, Mr. Alemayehu Wolde, and Mr. Benjamin Ongeri for their contribution to this work.

\section{Declaration}

This manuscript is original and the work has not been published elsewhere.

\section{Funding source}

This activity was funded by the MoHSS, with technical assistance from the USAID-funded Systems for Improved Access to Pharmaceuticals and Services Program, which is implemented in Namibia by Management Sciences for Health. The views and opinions expressed in this paper are those of the authors and not necessarily the views and opinions of the USAID. 


\section{Author details}

${ }^{1}$ Ministry of Health and Social Services, Windhoek, Namibia. ${ }^{2}$ Namibia Institute for Pathology, Windhoek, Namibia. ${ }^{3}$ Systems for Improved Access to Pharmaceuticals and Services, implemented by Management Sciences for Health, Windhoek, Namibia.

Received: 11 December 2012 Accepted: 30 April 2013

Published: 13 June 2013

\section{References}

1. Tunkel RA, Scheld WM: Pathogenesis and pathophysiology of bacterial meningitis. Clin Micro Rev 1993, 6:118-136.

2. Aronin SI: Bacterial meningitis: principles and practical aspects of therapy. Curr Infect Dis Rep 2000, 2:337-344.

3. O'Donnell EP, Hurt KM, Scheetz MH, Postelnick MJ, Scarsi KK: Empiric antibiotic selection for infectious emergencies: bacterial pneumonia, meningitis and sepsis. Drugs Today (Barc) 2009, 45(5):379-393.

4. Brouwer MC, Tunkel AR, Van de Beek D: Epidemiology, diagnosis, and antimicrobial treatment of acute bacterial meningitis. Clin Microbiol Rev 2010, 23(3):467-492

5. Geiseler PJ, Nelson KE, Levin S, Reddi KT, Moses VK: Community-Acquired Purulent meningitis: a review of 1,316 cases during the Antibiotic Era, 1954-1976. Rev Infect Dis 1980, 2(5):725-745. Sep-Oct.

6. Schuchat A, Robinson K, Wenger JD, Harrison LH, Farley M, Reingold AL, et al: Bacterial meningitis in the United States in 1995. Active Surveillance Team. N Engl J Med 1997, 337:970-976.

7. Davison KL, Ramsay ME: The epidemiology of acute meningitis in children in England and Wales. Arch Dis Child 2003, 88:662-664. http://www.ncbi. nlm.nih.gov/pmc/articles/PMC1719594/pdf/v088p00662.pdf

8. Jones ME, Draghi DC, Karlowsky JA, Sahm DF, Bradley JS: Prevalence of antimicrobial resistance in bacteria isolated from central nervous system specimens as reported by U.S. hospital laboratories from 2000 to 2002. Ann Clin Microbiol Antimicrob 2004, 3:3.

9. Grundmanna H, Klugman KP, Walsh T, Ramon-Pardoe P, Sigauquef B, Khanh W, et al: A framework for global surveillance of antibiotic resistance. Drug Resist Up 2011:1479-1487.

10. Kim KS: Treatment strategies for central nervous system infections. Expert Opin Pharmacother 2009, 10:1307-1317.

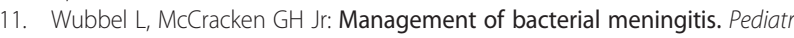
Rev 1998, 193:78-84

12. Kaplan SL: Clinical presentations, diagnosis, and prognostic factors of bacterial meningitis. Infect Dis Clin North Am 1999, 13:579-594.

13. Sutinen J, Sombrero L, Paladin FJ, Julkunen I, Leinikki P, Hernandez E, et al: Etiology of central nervous system infections in the Philippines and the role of serum C-reactive protein in excluding acute bacterial meningitis. Int J Infect Dis 1998-1999, 3(2):88-93.

14. Taylor WR, Nguyen $K$, Nguye D, Nguye $H$, Horby $P$, Nguyen $H L$, et al: The spectrum of central nervous system infections in an Adult Referral Hospital in Hanoi, Vietnam. PLoS One 2012, 7(8):e42099. August.

15. Huttunen $P$, Lappalainen M, Salo E, Lönnqvist T, Jokela P, Hyypiä T, Peltola $H$ : Differential diagnosis of acute Central Nervous System Infections in children using Modern Microbiological methods. Acta Paediatr 2009, 98(8):1300-1306.

16. Tunkel AR, Hartman BJ, Kaplan SL, Kaufman BA, Roos KL, Scheld WM, et al: Practice guidelines for the management of bacterial meningitis. Clin Infect Dis 2004, 39(9):1267-1284.

17. Fauci, AS, Lane, HC: HIV neurology. In: Hauser SL, Josephson SA, editors. Harrison's Neurology in Clinical Medicine. 2nd ed. New York, NY: McGraw-Hill Professional; 2010. p. 493-506.

18. Cunha BA: Effective antibiotic-resistance control strategies. Lancet 2001 357(9265):1307-1308.

19. Song JH, Jung SI, Ko KS, Kim NY, Son JS, Chang HH, et al: High prevalence of antimicrobial resistance among clinical Streptococcus pneumoniae isolates in Asia (an ANSORP study). Antimicrob Agents Chemother 2004, 48(6):2101-2107.

20. Centers for Disease Control and Prevention: Active Bacterial Core Surveillance Report, Emerging Infections Program Network, Streptococcus pneumoniae, 2010. 2012. http:/www.cdc.gov/abcs/reports-findings/survreports/spneu08.pdf.
21. Sarojamma V, Ramakrishna V: Prevalence of ESBL-Producing Klebsiella pneumoniae Isolates in Tertiary Care Hospital International Scholarly Research Network ISRN Microbiology Volume. 2011. Article ID 318348, 5 pages.

22. Sidjabat H, Nimmo GR, Walsh TR, Binotto E, Htin A, Hayashi Y, et al: Carbapenem resistance in Klebsiella pneumoniae due to the New Delhi Metallo-ß-lactamase. Clin Infect Dis 2011, 52(4):481-484.

doi:10.1186/2052-3211-6-4

Cite this article as: Mengistu et al:: Antimicrobial sensitivity patterns of cerebrospinal fluid (CSF) isolates in Namibia: implications for empirical antibiotic treatment of meningitis. Journal of Pharmaceutical Policy and Practice 2013 6:4.

\section{Submit your next manuscript to BioMed Central and take full advantage of:}

- Convenient online submission

- Thorough peer review

- No space constraints or color figure charges

- Immediate publication on acceptance

- Inclusion in PubMed, CAS, Scopus and Google Scholar

- Research which is freely available for redistribution

Submit your manuscript at www.biomedcentral.com/submit
C Biomed Central 